\title{
Predicting Slowdown for Networked Workstations
}

\author{
Silvia M. Figueira* and Francine Berman** \\ Department of Computer Science and Engineering \\ University of California, San Diego \\ La Jolla, CA 92093-0114 \\ \{silvia,berman\}@cs.ucsd.edu \\ http://www-cse.ucsd.edu/users/\{ silvia,berman \}
}

\begin{abstract}
Most applications share the resources of networked workstations with other applications. Since system load can vary dramatically, allocation strategies that assume that resources have a constant availability and/or capability are unlikely to promote performance-efficient allocations in practice. In order to best allocate application tasks to machines, it is critical to provide a realistic model of the effects of contention on application performance. In this paper, we present a model that provides an estimate of the slowdown imposed by competing load on applications targeted to highperformance clusters and networks of workstations. The model provides a basis for predicting realistic communication and computation costs and is shown to achieve good accuracy for a set of scientific benchmarks commonly found in high-performance applications.
\end{abstract}

\section{Introduction}

In the last decade, networks of workstations have emerged as powerful platforms for executing high-performance parallel applications. For such applications, the development of a performance-efficient allocation of tasks to machines is dependent upon a realistic prediction of application behavior under changing system load. In particular, additional applications executing on the system can dramatically affect the availability and capability of resources and must be factored into predictions of application execution costs. If an effective predictive model can be developed for time-shared multi-user environments, applications can be allocated in a way that promotes execution performance in the system.

In recent literature, machine workload has been used to parameterize the allocation of tasks to workstations in a network. However, many allocation strategies do not consider load characteristics in the measurement of workload (e.g., [1, 2, 4, 6]). When load characteristics are considered, the system environment for each workstation is frequently limited to at most one compute-intensive task and local tasks, which alternate idle with compute-

\footnotetext{
* Supported by a scholarship from CAPES and UFRJ (Brazil).

** Supported in part by NSF contract number ASC-9301788.

Copyright 1997 IEEE. Published in the Proceedings of the High Performance Distributed Computing Conference, August 5-8, 1997, Portland, OR. Personal use of this material is permitted. However, permission to reprint/republish this materia for advertising or promotional purposes or for creating new collective works for resale or redistribution to servers or lists, or to reuse any copyrighted component of this work in other works, must be obtained from the IEEE. Contact: Manager, Copyrights and Permissions / IEEE Service Center / 445 Hoes Lane / P.O. Box 1331 / Piscataway, NJ 08855-1331, USA. Telephone: + Intl. 908-562-3966.
}

intensive cycles $[10,15]$.

We believe that a model to predict contention effects on application performance must reflect both system characteristics and workload behavior. The dependence on workload behavior reflects the fact that different applications utilize different types of resources, and the requirements for these resources vary during the execution of each application.

In [8], we proposed a contention model based on system characteristics and workload behavior for twomachine networks. In this paper, we develop a contention model for application performance on high-performance clusters and networked workstations. Our model is based on the evaluation of a slowdown factor, which is used to predict computation and communication costs in a timeshared multi-user networked environment. The parameters required for our models are detailed enough to allow good accuracy, but reasonable to obtain or calculate at run-time.

This paper is organized as follows. Section 2 introduces our approach. In Section 3, we analyze contention effects in high-performance clusters. Section 4 discusses contention effects in workstation networks. Section 5 evaluates the proposed model. Section 6 concludes with a summary and future work.

\section{Approach}

In the following sections, we define a contention measure, the slowdown factor, to adjust the computation times and communication costs of an application to accommodate for system load. In a networked system, application tasks may be assigned to distinct execution sites to promote concurrent execution and/or to take advantage of distributed data sources, aggregate memory, or I/O bandwidth. If slowdown can be defined accurately, it can be utilized to produce more realistic predictions of application performance. Such predictions form the foundation of a performance-efficient strategy for scheduling. We model slowdown for the time required to compute an application task at a single execution site in the system, and for the time required for communication between distinct execution sites.

Define $X_{m \text {,ded }}$ as the time to execute task $X$ on machine $m$ in dedicated mode, and $\left(X_{m} \rightarrow Y_{n}\right)_{\text {ded }}$ as the cost to transfer data between task $X$ and task $Y$ when task $X$ 
executes on machine $m$ and task $Y$ executes on machine $n$, in dedicated mode. Since computation and communication costs are affected by contention for resources on both machines and on the communication link, the time to execute task $X$ on machine $m$ is given by

$$
X_{m}=X_{m, \text { ded }} \times s d_{m},
$$

where $s d_{m}$ is a slowdown factor dependent on the load in machine $m$. The cost to transfer data between task $X$ and task $Y$, when task $X$ executes on machine $m$ and task $Y$ executes on machine $n$, is

$$
X_{m} \rightarrow Y_{n}=\left(X_{m} \rightarrow Y_{n}\right)_{d e d} \times s d_{m \rightarrow n},
$$

where $s d_{m \rightarrow n}$ is a slowdown factor dependent both on the traffic between machine $m$ and machine $n$ and on contention in both machines.

Equations (1) and (2) demonstrate how slowdown factors can be used to predict execution time in the presence of contention. The following sections show how to derive these slowdown factors for two different platforms: high-performance clusters and workstation networks.

We make the following assumptions: We assume applications to be coarse-grained, scientific programs that are basically CPU-bound. We assume that the applications executing on each workstation fit in memory, or at least that their working sets fit in memory, so that no delay is imposed by extra swapping caused by lack of memory space. We also assume multiple applications to have the same priority as one another and to be scheduled locally in round-robin fashion on the time-shared systems. In practice, most operating systems executing on workstations employ a priority-based scheduling strategy that reduces to a round-robin policy when the executing applications are CPU-bound [7]. Since we assume the applications to be coarse-grained, considering a roundrobin local scheduler for time-shared systems is a reasonable assumption.

We assume that communication between machines involves the transference of large bursts of data, requires data conversion, and uses TCP/IP via sockets.

Our calculation of slowdown factors currently requires information about all the applications executing on the system, i.e., we assume that information about the load is available or is easily obtainable. This is a reasonable assumption for local environments (e.g., distributed facilities at the San Diego Supercomputer Center or the UCSD Parallel Computation Laboratory), where such information is typically available. Determining slowdown factors, when only partial information about contending applications is known, is an open question and part of our research plans.

Finally, we assume that the time to execute a task in dedicated (single-user) mode is known or has been calculated previously. The contribution of this research is to use dedicated time estimates and slowdown factors to provide reasonably accurate estimates of application behavior in a time-shared multi-user system.
To validate our models we have used scientific computations on systems in which contention is generated by synthetic loads. This guarantees equivalent conditions for comparative experiments. In this paper, we demonstrate our model on serial versions of three benchmarks commonly used in scientific applications: an SOR benchmark [5], which solves Laplace's equation and was developed using PVM [13], a Matrix Multiplication benchmark [11], which was developed using KeLP [9] and MPI [12], and a Multigrid benchmark [5], which was also developed using KeLP and MPI. We have verified the model with various load conditions. In the experiments performed, contention was generated by a synthetic load generator in which computation and communication cycles alternate. The load was parameterized so that the duration of the computation cycle, the direction of the communication, and the number of messages and message size in a communication burst, as well as other parameters could be varied.

\section{Contention Effects in High-Performance Clusters}

Consider a set of homogeneous workstations connected by a dedicated high-speed network. Examples of this type of high-performance cluster include the DEC Alpha-Farm and the IBM SP-2. We consider each node in the cluster as an individual machine and calculate the slowdown on computation and communication costs as $s d_{a}$ and $s d_{a \rightarrow b}$, where $a$ and $b$ are two nodes in the cluster.

In a cluster, applications executing on a machine may be communicating with various machines. The delay imposed by communication activity on both computation and communication costs may vary with the bandwidth available on the links used by the competing applications. This happens because the bandwidth available reflects the amount of CPU used for communication.

We demonstrate this phenomenon using two nodes of a DEC Alpha-Farm ${ }^{1}$, which we call $\mathrm{m}_{1}$ and $\mathrm{m}_{2}$. Figure 1 shows the difference between the delay imposed on computation at node $m_{1}$ by one application communicating from node $m_{1}$ to node $m_{2}$ when different bandwidths are available. The graph shows curves for execution of the SOR benchmark with different problem sizes (given by $N \times N$ ) subject to no contention, and subject to contention when 1.55 and $3.21 \mathrm{Mwords} /$ second are available. The communicating process is the same for both experiments. The bandwidth available reflects the load on the communication link during the experiments and was calculated with a ping-pong benchmark. This benchmark transferred $1 \mathrm{~K}$ messages with $1 \mathrm{~K}$ words each from one machine to the other. It is clear that when more bandwidth is available, the communicating process uses more CPU, and the delay it imposes on the SOR benchmark is greater.

\footnotetext{
1. The DEC Alpha-Farm used is a cluster of eight DEC Alpha 3000/400 workstations located at the San Diego Supercomputer Center. The Alphas are connected via a dedicated GIGAswitch. All experiments in this section were performed on this platform.
} 


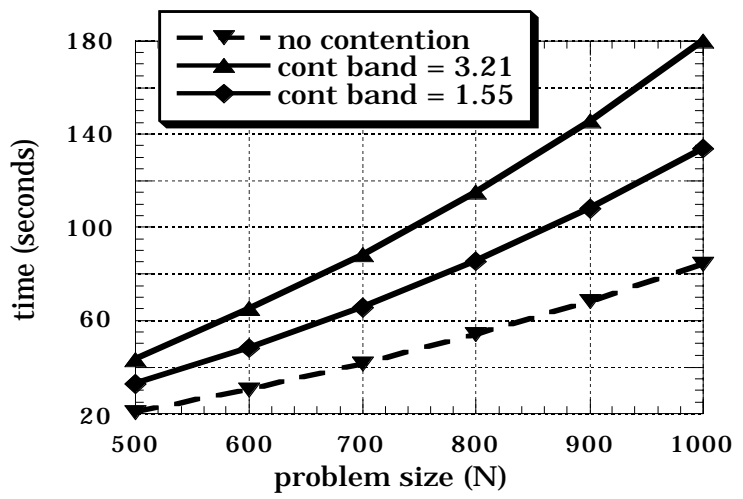

Figure 1: Time to execute the SOR benchmark on one node of the DEC Alpha-Farm in dedicated mode and multiuser mode, competing for the CPU with one communication-bound process when different bandwidths are available.

The slowdown imposed by communication varies with the communicating machines and the direction of the transferences. Note that the effects caused by the direction and target of communication are reflected in bandwidth variation. We will use this bandwidth measure as a parameter for both computation and communication slowdown.

\subsection{Computation Slowdown in High-Performance Clusters}

The previous experiment indicates that computation on each node is affected by other applications executing on the same node. The delay imposed by additional applications computing on a node reflects the fact that CPU time is evenly split among the competing processes. (Recall that we assume that the node scheduling policy is round-robin.) The delay imposed by additional applications communicating depends on the number of applications communicating, on the bandwidth available, and on the size of the messages being transferred. The impact of the message size is reflected in the bandwidth variation. Hence we can approximate the slowdown imposed on a task executing on node $a$ as:

$$
\begin{aligned}
s d_{a}=1 & +\sum_{i=1}^{p_{a}}\left(p p_{a, i} \times i\right) \\
& +\sum_{i=1}^{p_{a}}\left(p m_{a, i} \times \text { delay }_{\text {comm }}^{i, k}\right),
\end{aligned}
$$

where $p_{a}$ is the number of additional applications executing on node $a, p p_{a, i}$ is the probability that $i$ applications will compute on node $a$ at the same time, $p_{a, i}$ is the probability that $i$ applications on node $a$ will try to communicate at the same time, and delay $y_{\text {comm }}^{i, k}$ is the delay imposed on computation by $i$ communicating applications when the average bandwidth available is $k$ Mwords/second.
In the slowdown calculation, the first summation accounts for additional applications competing for CPU cycles, which are evenly split among all CPU-bound applications on the node. The second summation accounts for additional communicating applications. The values $p p_{a, i}$ and $p m_{a, i}$ are calculated based on the percentages of computation and communication associated with each application executing on node $a$.

The value delay $y_{\text {comm }}^{i, k}$ is the average delay imposed on computation by $i$ communication-intensive applications executing on the node. It is calculated as the average delay imposed on a CPU-bound application by $i$ contention generators that transfer messages to another node when the bandwidth available is $k$ Mwords/second. Since the delay imposed by receiving and sending applications is generally roughly the same, we use delay comm in both situations.

The value delay comm is system-dependent and does not change dynamically. Our experiments determined delay $\begin{gathered}i, k \\ \text { comm }\end{gathered}$ as a set of functions of $k$, each of which is indexed by a different value of $i$. The functions are determined just once for each platform and, therefore, their calculation does not impose any additional overhead on the scheduling process at run-time. Each function is determined with a two-piece linear regression on the numbers obtained by a benchmark. The benchmark measures the delay imposed on a CPU-bound application by $i$ communication-bound applications that communicate with other nodes under decreasing bandwidth. At runtime, the value for $k$ - the average bandwidth available on the links used by competing applications - can be predicted with statistical methods (the Network Weather Service $[3,14]$, for example) and used to determine delay $\begin{gathered}i, k \\ \text { comm }\end{gathered}$.

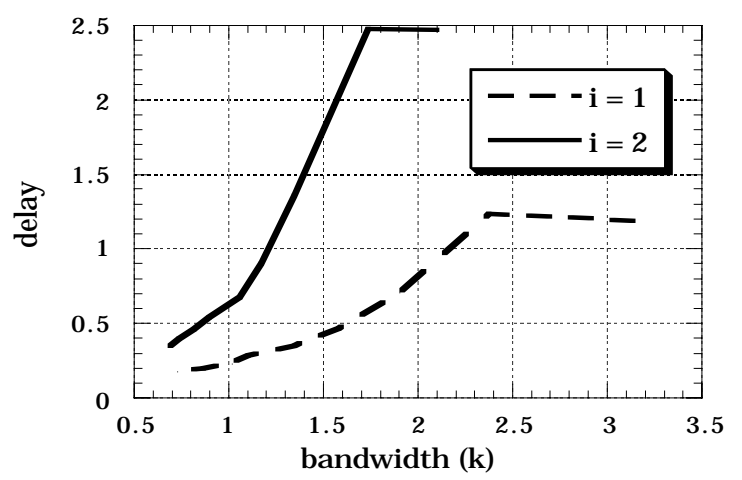

Figure 2: Graph of delay $y_{\text {comm }}^{i, k}$ for $i=1$ and $i=2$. Each curve in the graph provides the value for the respective delay, which varies with the bandwidth $(k)$.

Figure 2 shows the curves obtained for delay comm, when $i=1$ and $i=2$. In low-bandwidth systems, delay ${ }_{\text {comm }}^{i, k}$ may be approximated by just one of the indexed curves or by a single value. One curve is enough when the delay does not vary with $i$, whereas a single value is enough when the range of the delay imposed by 
communication is small, as described in Section 4.1.

The slowdown factor is calculated at run-time and, therefore, it is important to guarantee that its calculation is efficient so that it does not impose too much overhead on the scheduling process. Although the values $p p_{a, i}$ and $p m_{a, i}$ change with the load on the system and are obtained at run-time, they can be calculated using dynamic programming in time $O\left(p_{a}{ }^{2}\right)$. Since $p_{a}$ is generally small and the overall calculation of the slowdown takes $O\left(p_{a}{ }^{2}\right)$ time, the overhead imposed by its calculation is negligible.

Figure 3 is a comparison of measured with predicted (modeled) execution times for SOR, when $s d_{a}$ is calculated using equation (3). The SOR benchmark was executed in multi-user and single-user modes for a variety of problem sizes. Multi-user mode was emulated by an additional application executing on the same node. This application alternates computation and communication cycles and communicates with another node $45 \%$ of the time. The bandwidth available in the system during the experiment was 1.79 Mwords/second, and the slowdown imposed by the additional application was 1.56 . In this example, our predictions were within an average error of $3 \%$ of the actual measurements.

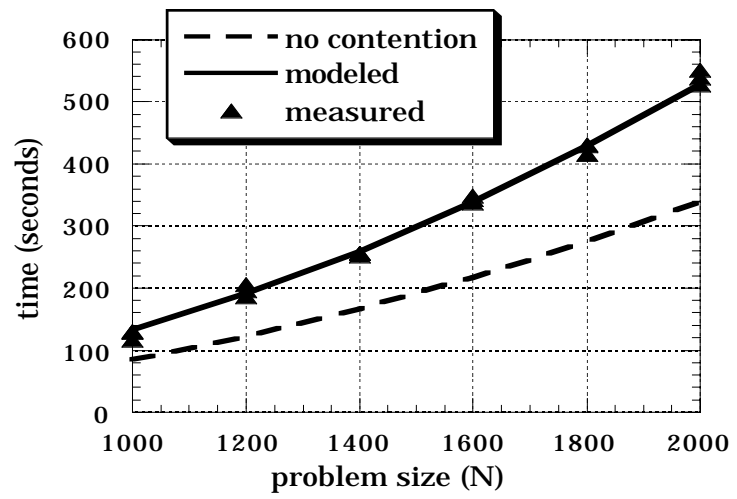

Figure 3: Time to execute the SOR benchmark on one node of the DEC Alpha-Farm in dedicated mode and competing with one application.

This experiment illustrates how slowdown can be used to "correct" single-user predictions to achieve multi-user predictions.

To validate our model, we ran a serial (one processor) version of the benchmarks with diverse configurations of one, two and three instances of the contention generator. These configurations were defined by the contention generator parameters. Table 1 shows experiments with a serial version of the Multigrid benchmark and two competing applications, and Table 2 shows experiments with a serial version of the Matrix Multiply benchmark. Each row in the tables corresponds to one execution of the benchmark with five different problem sizes. The average error column indicates, for each experiment, how far our predictions of execution time were from measured execution times. As the tables show, we varied both the burst size used by the competing application and the average bandwidth available on the links used by the competing applications.

Table 1: Experiments with Multigrid

\begin{tabular}{|c|c|c|}
\hline $\begin{array}{c}\text { Burst Sizes (number of } \\
\text { messages / message size } \\
\text { in words) per } \\
\text { Competing Application }\end{array}$ & $\begin{array}{c}\text { Average } \\
\text { Bandwidth } \\
\text { (Mwords/sec) }\end{array}$ & $\begin{array}{c}\text { Average } \\
\text { Error }\end{array}$ \\
\hline \hline $10 / 1000,10 / 1000$ & 2.75 & $2 \%$ \\
\hline $1000 / 1000,1000 / 1000$ & 2.75 & $1 \%$ \\
\hline $5000 / 1000,5000 / 1000$ & 2.75 & $1 \%$ \\
\hline $100 / 1000,100 / 1000$ & 2.12 & $11 \%$ \\
\hline $1000 / 1000,1000 / 1000$ & 2.12 & $9 \%$ \\
\hline $5000 / 1000,5000 / 1000$ & 2.12 & $4 \%$ \\
\hline
\end{tabular}

Table 2: Experiments with Matrix Multiply

\begin{tabular}{|c|c|c|}
\hline $\begin{array}{c}\text { Burst Sizes (number of } \\
\text { messages / message size } \\
\text { in words) per } \\
\text { Competing Application }\end{array}$ & $\begin{array}{c}\text { Average } \\
\text { Bandwidth } \\
\text { (Mwords/sec) }\end{array}$ & $\begin{array}{c}\text { Average } \\
\text { Error }\end{array}$ \\
\hline \hline $4000 / 4000$ & 1.51 & $5 \%$ \\
\hline $2000 / 2000,2000 / 2000$ & 3.18 & $1 \%$ \\
\hline $\begin{array}{c}2000 / 2000,3000 / 3000 \\
4000 / 4000\end{array}$ & 3.89 & $9 \%$ \\
\hline $2000 / 1000,2000 / 1000$ & 2.45 & $6 \%$ \\
$2000 / 1000$ & & \\
\hline
\end{tabular}

\subsection{Communication Slowdown in High-Performance Clusters}

In the previous subsection, we developed a formula for $s d_{a}$, the slowdown on computation at processor $a$ due to contention. In this subsection, we develop a formula for $s d_{a \rightarrow b}$, the slowdown on communication.

In high-performance clusters, when nodes are connected by switches and contention for the interconnection network is negligible, communication delay between two nodes is affected primarily by other applications on the two nodes. These applications may be computing, or communicating within the source and destination nodes, or communicating with other nodes.

Our experiments show that various factors affect communication costs. The number of communicating and computing applications executing on the source and destination nodes, the size of the messages being transferred by the communicating applications, the bandwidth available on the links used, and the destination and direction of the communication, all affect the amount of delay.

We use Figure 4 to illustrate the fact that the bandwidth available in the links used by competing applications affects the delay imposed by these applications on communication. Suppose we want to measure communication costs from node $\mathrm{m}_{1}$ to node $\mathrm{m}_{2}$. If node 
$\mathrm{m}_{2}$ hosts an application that is communicating with node $\mathrm{m}_{3}$, the bandwidth available on this link (between nodes $\mathrm{m}_{2}$ and $\mathrm{m}_{3}$ ) will affect the amount of CPU required from nodes $\mathrm{m}_{2}$ and $\mathrm{m}_{3}$ to perform this communication and, consequently, communication costs between nodes $m_{1}$ and $\mathrm{m}_{2}$.

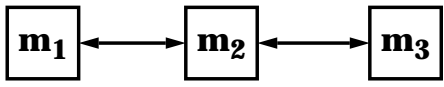

Figure 4: Communication activity among 3 nodes in a dedicated cluster.

Figure 5 shows the difference between the delay imposed on communication from node $m_{1}$ to node $m_{2}$ by one competing application that transfers data from node $\mathrm{m}_{2}$ to node $\mathrm{m}_{3}$ under different bandwidths. The graph shows the time to transfer bursts of 1000 messages in three different situations: when there is no contention on the source and destination nodes; when a competing application is also executing and the bandwidth available between nodes $\mathrm{m}_{2}$ and $\mathrm{m}_{3}$ is 2.23 Mwords/second, and when the competing application is also executing but the bandwidth available between nodes $\mathrm{m}_{2}$ and $\mathrm{m}_{3}$ is 1.20 Mwords/second. The competing application is the same for both experiments.

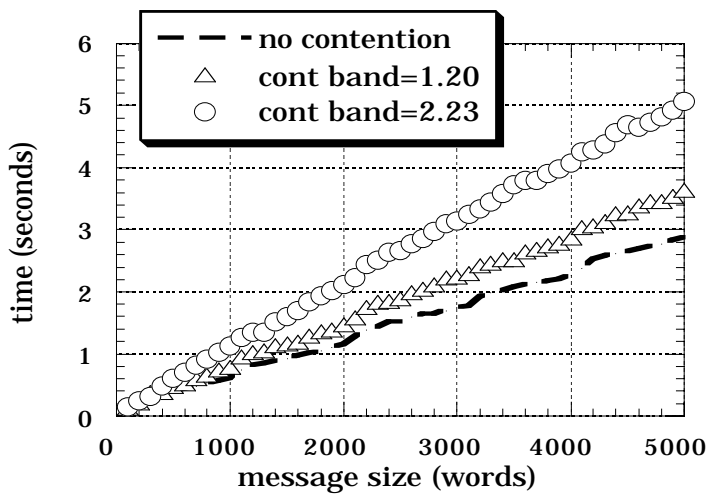

Figure 5: Time to transfer bursts with 1000 messages between two nodes of the DEC Alpha-Farm in dedicated mode and competing with a communication-bound process that transfers messages through a link where different bandwidths are available.

A prediction of the bandwidth available on all the links used by competing applications is required at run-time so that the delay imposed on communication can be calculated. However, we can achieve good results using a simpler model that is based just on the bandwidth available between nodes $a$ and $b$. Using this model we calculate the slowdown on communication as follows:

$$
s d_{a \rightarrow b}=\frac{i b_{a \rightarrow b}}{c b_{a \rightarrow b}},
$$

where $i b_{a \rightarrow b}$ is the initial bandwidth, or bandwidth available when $\left(X_{a} \rightarrow Y_{b}\right)$ ded was calculated, and $c b_{a \rightarrow b}$ is the current bandwidth, or bandwidth available at run-time.

Equation (4) reflects the delays imposed by both computation and communication activities on communication costs. Although these activities do not appear in the formula, they are embedded in the current bandwidth measure, $c b_{a \rightarrow b}$, which accounts for competition for both CPU and communication link.

The value for $i b_{a \rightarrow b}$ can be calculated with a benchmark whenever $\left(X_{a} \rightarrow Y_{b}\right)$ ded is calculated. The benchmark sends 1024 messages with 1024 words each from one node to another and waits for the answer.

The value for $c b_{a \rightarrow b}$ can be obtained at run-time with statistical methods employed by performance prediction tools, such as the Network Weather Service [3, 14]. In our experiments, since the contention in the cluster is generated by a synthetic load, we determined the value for $c b_{a \rightarrow b}$ by measuring the bandwidth at run-time with the same benchmark used to calculate $i b_{a \rightarrow b}$.

Figure 6 illustrates communication formula (4). It shows the time for transferring bursts of 1000 equal-sized messages between two nodes in dedicated time $\left(i b_{a \rightarrow b}=\right.$ $6.21 \mathrm{Mwords} / \mathrm{second}$ ), as well as modeled and actual times for transferring the same messages between the two nodes when $c b_{a \rightarrow b}=3.67$ Mwords/second. The error in this experiment was within $9 \%$ on average.

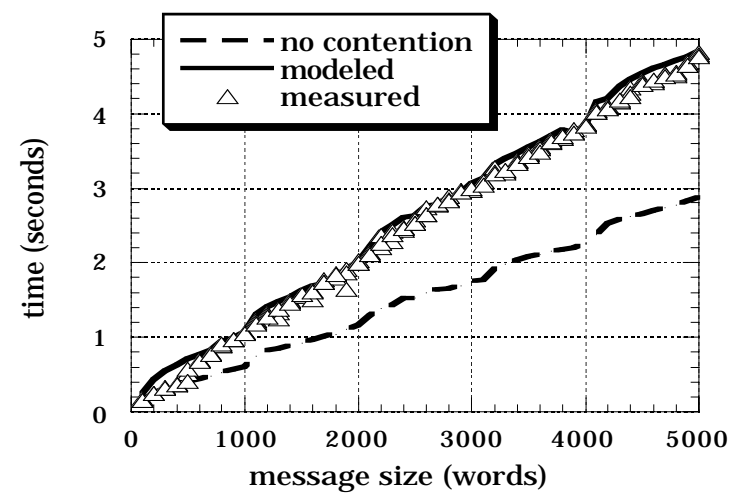

Figure 6: Time to transfer bursts with 1000 messages each between two nodes, in dedicated mode and under decreased bandwidth.

Figure 7 also illustrates formula (4). It shows the time for transferring bursts of 2000 equal-sized messages between two nodes in dedicated time $\left(i b_{a \rightarrow b}=6.21\right.$ Mwords/second), as well as modeled and actual times for transferring the same messages between the two nodes when $c b_{a \rightarrow b}=1.95 \mathrm{Mwords} / \mathrm{second}$. The error in this experiment was within $13 \%$ on average.

To validate the model shown in formula (4), we ran a ping-pong benchmark (which transfers bursts of samesized messages) with diverse configurations of one, two and three instances of the contention generator. These configurations are indexed by the contention generator parameters. Table 3 presents a representative set of the experiments performed to verify the model. Each row of the table corresponds to three executions of the ping-pong benchmark for each message size, which grows in steps of 100 words. The average error column indicates, for each 
experiment, how far our predictions of communication costs (using $s d_{a \rightarrow b}$ ) were from the actual communication measurements. As the table shows, the bandwidth available and burst size of the ping-pong benchmark were varied during the experiments.

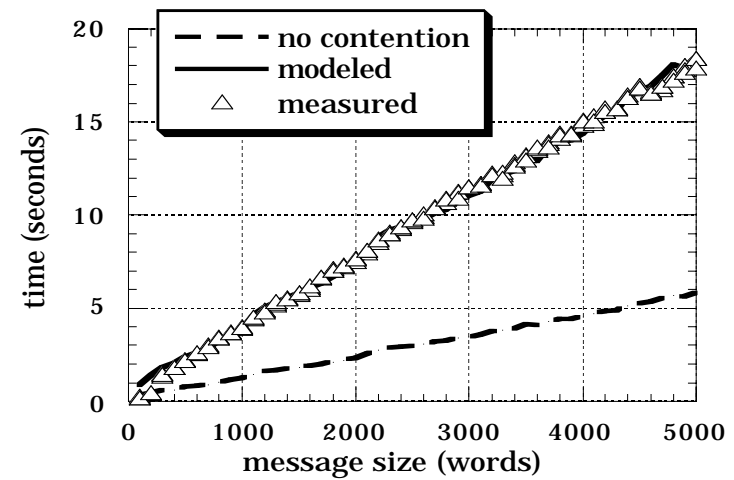

Figure 7: Time to transfer bursts with 2000 messages each between two nodes, in dedicated mode and under decreased bandwidth.

Table 3: Experiments with Communication

\begin{tabular}{|c|c|c|}
\hline $\begin{array}{l}\text { Burst Sizes (number of } \\
\text { messages / message size } \\
\text { in words) }\end{array}$ & $\begin{array}{c}\text { Average } \\
\text { Bandwidth } \\
\text { (Mwords/sec) }\end{array}$ & $\begin{array}{c}\text { Average } \\
\text { Error }\end{array}$ \\
\hline $100 / 100-1000$ & 1.19 & $368 \%$ \\
\hline $100 / 100-1000$ & 2.32 & $151 \%$ \\
\hline $100 / 100-1000$ & 3.44 & $78 \%$ \\
\hline $100 / 1000-3000$ & 1.19 & $16 \%$ \\
\hline $100 / 1000-3000$ & 2.32 & $51 \%$ \\
\hline $100 / 1000-3000$ & 3.44 & $23 \%$ \\
\hline $1000 / 100-1000$ & 1.19 & $81 \%$ \\
\hline $1000 / 100-1000$ & 2.32 & $42 \%$ \\
\hline $1000 / 100-1000$ & 3.44 & $33 \%$ \\
\hline $1000 / 1000-3000$ & 1.19 & $14 \%$ \\
\hline $1000 / 1000-3000$ & 2.32 & $4 \%$ \\
\hline $1000 / 1000-3000$ & 3.44 & $2 \%$ \\
\hline $5000 / 100-1000$ & 1.19 & $57 \%$ \\
\hline $5000 / 100-1000$ & 2.32 & $20 \%$ \\
\hline $5000 / 100-1000$ & 3.44 & $16 \%$ \\
\hline $5000 / 1000-3000$ & 1.19 & $10 \%$ \\
\hline $5000 / 1000-3000$ & 2.32 & $3 \%$ \\
\hline $5000 / 1000-3000$ & 3.44 & $2 \%$ \\
\hline
\end{tabular}

According to the results shown in Table 3, the error increases when the burst size is small and the available bandwidth is low. This happens because, in this highbandwidth platform, short bursts are not sensitive to bandwidth variations. A benchmark that calculates the bandwidth using a tailored burst size will overcome this problem. This is illustrated in Table 4, which shows the slowdown produced with a benchmark that measures the bandwidth using a specific burst size. For short bursts, the slowdown is approximately 1 , whereas for larger bursts, it is approximately 4 (assuming the same load in the network).

Table 4: Experiments with Bandwidth Benchmarks

\begin{tabular}{|c|c|}
\hline $\begin{array}{c}\text { Burst Size (number of } \\
\text { messages / message size in } \\
\text { words) used by the Benchmark }\end{array}$ & $s d_{a \rightarrow b}$ \\
\hline \hline $100 / 100$ & 1.08 \\
\hline $100 / 500$ & 0.96 \\
\hline $1000 / 500$ & 3.99 \\
\hline $1024 / 1024$ & 3.97 \\
\hline $5000 / 500$ & 3.96 \\
\hline
\end{tabular}

\section{Contention Effects in}

\section{Workstation Networks}

The slowdown factors calculated in the previous section were developed for high-performance clusters in which the network connecting the nodes was dedicated to the nodes, and non-negligible bandwidth variations were caused exclusively by applications on the cluster. In this section, we extend the model to reflect a more general scenario of a workstation network (such as a computer laboratory) in which machines may belong to different networks and are connected by non-dedicated network links. The basic differences between a workstation network and a high-performance cluster are:

- Less bandwidth is typically available in workstation networks.

- In workstation networks, machines may not all be the same.

- Links connecting the machines in a workstation network may not have the same bandwidth.

- Bandwidth between machines in a workstation network may be affected by applications executing on other machines that share network links with the machines in the network.

It is important to note that, in this platform, we use the term communication link to refer to a connection between two nodes that may or may not be directly connected by a physical network link.

We consider each node in the workstation network to be an individual machine. In this section, we calculate the slowdown for computation and communication costs, $s d_{a}$ and $s d_{a \rightarrow b}$ respectively, where $a$ and $b$ are two nodes in the network.

We demonstrate our results using the four nodes of a workstation network consisting of two DEC Alphas ${ }^{2}$ (located in the Computer Systems Laboratory at UCSD) connected to one another by Ethernet and two IBM RS-

2. The DEC Alphas used are DEC Alpha 3000/800 workstations. 
6000s (located in the Parallel Computation Laboratory at UCSD) also connected by Ethernet. Each pair of workstations is connected via Ethernet and is part of a non-dedicated network.

\subsection{Computation Slowdown in Workstation Networks}

Computation on each node is affected by additional applications executing on the same node, which may be computing or communicating with another node. The delay imposed by applications computing on the node reflects the fact that CPU time is evenly split among the competing processes. As before, delay imposed on computation by communicating applications depends on the variation of the bandwidth on the links used. However, since the bandwidth is typically lower for the workstation network (in comparison with the high-performance cluster), the range of its variation is small, as is the range of the variation on the delay. For this reason, an average delay can be used to approximate the delay imposed by communication on computation. Consequently, the slowdown imposed on a computation executing on node $a$ can be modeled by

$$
\begin{aligned}
s d_{a}=1 & +\sum_{i=1}^{p_{a}}\left(p p_{a, i} \times i\right) \\
& +\sum_{i=1}^{p_{a}}\left(p_{a, i} \times \text { delay }_{\text {comm }}\right),
\end{aligned}
$$

where $p_{a}$ is the number of extra applications executing on the node, $p p_{a, i}$ is the probability that $i$ applications will compute on node $a$ at the same time, $p m_{a, i}$ is the probability that $i$ applications on node $a$ will try to communicate at the same time, and delay ${ }_{\text {comm }}$ is the delay imposed on computation by communication activity.

As before, the first summation accounts for other applications competing for CPU cycles, which are evenly split among CPU-bound applications on the node. The second summation accounts for computation required to support the communication of other applications. Both $p p_{a, i}$ and $p m_{a, i}$ are calculated based on the percentages of computation and communication associated with each application executing on node $a$.

The value delay $y_{\text {comm }}$ is the average delay imposed on computation by communicating applications executing on the node. It is the average of two delays. The first is the maximum delay imposed by communication on computation. This can be calculated by measuring the delay imposed on a CPU-bound application by a communication-intensive application that communicates with the "closest" machine in the workstation network transferring messages with 1000 words. The closest machine is the one with which the node can communicate with highest bandwidth (generally the closest machine is one located on the same physical network, e.g., on the same Ethernet). The second is the minimum delay imposed by communication on computation, which generally tends to 0 because the delay decreases with the bandwidth available.

The slowdown factor $s d_{a}$ is calculated at run-time and, therefore, it is important to guarantee that its calculation is efficient so that it does not impose excessive overhead on the scheduling process. The value delay $y_{\text {comm }}$ is systemdependent and does not change dynamically. It is calculated just once for each platform and, therefore, its calculation does not impose any additional overhead on the scheduling process at run-time. Although the values $p p_{a, i}$ and $p m_{a, i}$ change with the load on the system and are obtained at run-time, they can be calculated as before by dynamic programming in time $O\left(p_{a}{ }^{2}\right)$. Since $p_{a}$ is small and the overall calculation of the slowdown takes $O\left(p_{a}{ }^{2}\right)$ time, the overhead imposed by its calculation is negligible.

Figure 8 shows the modeled and measured times for executing the SOR benchmark on a DEC Alpha in dedicated (single-user) and non-dedicated (multi-user) modes, parameterized by problem size (which is $N \times N$ ). In this experiment, two additional applications are executing on the machine. They alternate computation and communication cycles and communicate with another machine $24 \%$ of the time. In this example, our predictions were within an average error of $6 \%$ of the actual measurements.

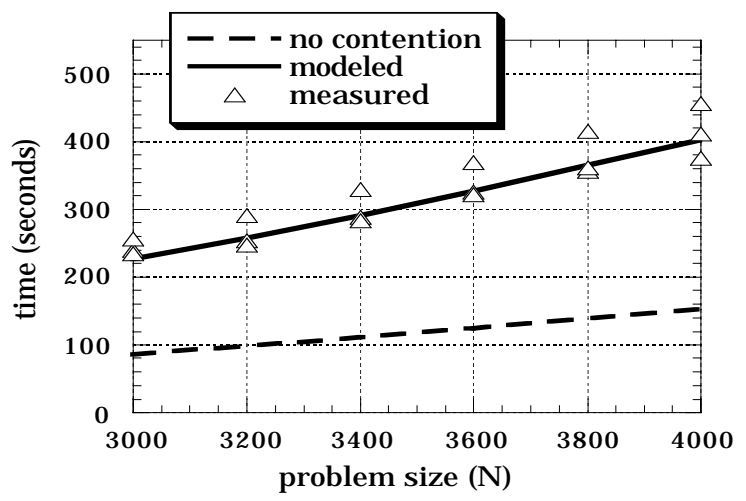

Figure 8: Time to execute the SOR algorithm on a DEC Alpha, in dedicated and non-dedicated mode, competing with two extra applications. The contending applications communicate $24 \%$ of the time and compute the remaining time.

To validate the model described in equation (5), we ran a serial version of the SOR benchmark and other applications with diverse configurations of one, two and three instances of the contention generator. These configurations were defined by the contention generator parameters. Table 5 presents experiments performed with a serial version of the SOR benchmark when there are competing applications on the node. Each row of the table corresponds to one execution of the benchmark (on a DEC Alpha) with six different problem sizes. The average error column indicates, for each experiment, how far our predictions were from the actual measurements of execution time. As the table shows, we vary the 
computation/communication ratio of the competing applications and the burst size used by the competing applications.

Table 5: Experiments with Computation

\begin{tabular}{|c|c|c|}
\hline $\begin{array}{c}\text { Amount(s) of } \\
\text { Communication } \\
\text { per } \\
\text { Competing } \\
\text { Application }\end{array}$ & $\begin{array}{l}\text { Burst Size(s) (number of } \\
\text { messages / message size } \\
\text { in words) } \\
\text { per Competing Application }\end{array}$ & $\begin{array}{l}\text { Average } \\
\text { Error }\end{array}$ \\
\hline $24 \%$ & $1000 / 2000$ & $5 \%$ \\
\hline $49 \%$ & $2000 / 1000$ & $9 \%$ \\
\hline $58 \%$ & $1000 / 3000$ & $9 \%$ \\
\hline $44 \%$ & $5000 / 1000$ & $7 \%$ \\
\hline $15 \%$ & $5000 / 100$ & $8 \%$ \\
\hline $14 \%$ & $100 / 5000$ & $3 \%$ \\
\hline $49 \%, 49 \%$ & $2000 / 1000,2000 / 1000$ & $6 \%$ \\
\hline $15 \%, 14 \%$ & $5000 / 100,100 / 5000$ & $8 \%$ \\
\hline $38 \%, 15 \%$ & $1000 / 2000,5000 / 100$ & $13 \%$ \\
\hline $48 \%, 48 \%$ & $1000 / 2000,1000 / 2000$ & $3 \%$ \\
\hline $48 \%, 21 \%$ & $1000 / 2000,5000 / 100$ & $18 \%$ \\
\hline $58 \%, 32 \%$ & $1000 / 3000,1000 / 1000$ & $15 \%$ \\
\hline $49 \%, 49 \%, 49 \%$ & $\begin{array}{l}2000 / 1000,2000 / 1000, \\
2000 / 1000\end{array}$ & $6 \%$ \\
\hline $58 \%, 32 \%, 49 \%$ & $\begin{array}{c}1000 / 3000,1000 / 1000 \\
2000 / 1000\end{array}$ & $22 \%$ \\
\hline
\end{tabular}

\subsection{Communication Slowdown in Workstation Networks}

Our experiments show that various factors affect communication costs. The number of computing and communicating applications executing on the two nodes, the size of the messages being transferred by communicating applications, the bandwidth available on the links used, and the destination and direction of the communication, all affect the delay imposed on communication. As in the high-performance cluster case, bandwidth variation reflects all these factors and can be used in a simple model. Following the analysis for the high-performance clusters, slowdown on communication can be modeled by

$$
s d_{a \rightarrow b}=\frac{i b_{a \rightarrow b}}{c b_{a \rightarrow b}}
$$

as shown in Section 3.2.

Figure 9 illustrates communication formula (6). It shows the time for transferring bursts of 1000 equal-sized messages between the two IBM RS-6000s (located in the same subnet) when $i b_{a \rightarrow b}=0.91$ Mwords/second, and modeled and actual times for transferring the same bursts when there are additional applications alternating computation and communication cycles on the nodes. The value for $c b_{a \rightarrow b}$ is 0.33 Mwords/second, $s d_{a \rightarrow b}=2.76$, and the average error for modeled versus actual communication costs was within $7 \%$. The peak obtained by transferring messages with 3100 words reflects an inefficiency of the IBM RS-6000 system in dealing with this specific message size.

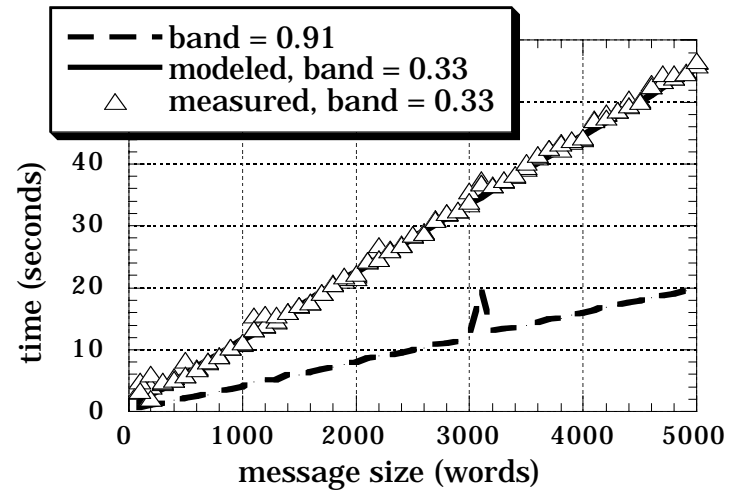

Figure 9: Time to transfer bursts with 1000 messages between machines in the same subnet.

Figure 10 also illustrates communication formula (6). It shows the time for transferring bursts of 2000 equal-sized messages between one IBM RS-6000 and one DEC Alpha in different subnets when $i b_{a \rightarrow b}=0.48$ Mwords/second, and modeled and actual times for transferring the same bursts when there are additional applications alternating computation and communication cycles on the nodes. The value for $c b_{a \rightarrow b}$ is 0.28 Mwords/second, $s d_{a \rightarrow b}=1.71$, and the average error for communication cost modeled with slowdown versus actual communication cost was within $7 \%$.

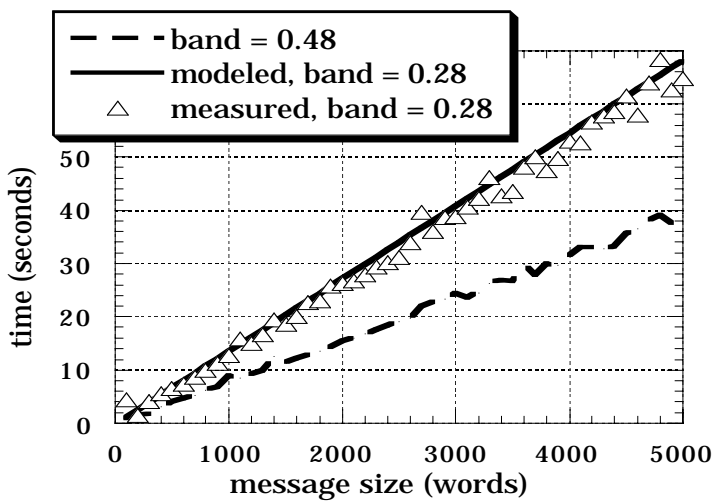

Figure 10: Time to transfer bursts with 1000 messages between machines in the different subnets.

To validate our model of communication slowdown, we ran a ping-pong benchmark (which transfers bursts of same-sized messages) with diverse configurations of one, two and three instances of the contention generator. These configurations were defined by the contention generator parameters. Table 6 presents a representative set of the experiments performed with the ping-pong benchmark. Each row of the table corresponds to three executions of the ping-pong benchmark for each message size, which grows in steps of 100 words. The average error column 
indicates, for each experiment, how far our predictions were from the actual measurements of communication costs. As the table shows, we vary the bandwidth available and burst size of the ping-pong benchmark.

Table 6: Experiments with Communication

\begin{tabular}{|c|c|c|}
\hline $\begin{array}{c}\text { Burst Sizes (number of } \\
\text { messages / message size } \\
\text { in words) }\end{array}$ & $\begin{array}{c}\text { Average } \\
\text { Bandwidth } \\
\text { (Mwords/sec) }\end{array}$ & $\begin{array}{c}\text { Average } \\
\text { Error }\end{array}$ \\
\hline \hline $10 / 100-1000$ & 0.48 & $23 \%$ \\
\hline $10 / 100-1000$ & 0.68 & $43 \%$ \\
\hline $10 / 1000-3000$ & 0.48 & $5 \%$ \\
\hline $10 / 1000-3000$ & 0.68 & $43 \%$ \\
\hline $1000 / 100-1000$ & 0.48 & $19 \%$ \\
\hline $1000 / 100-1000$ & 0.68 & $8 \%$ \\
\hline $1000 / 1000-3000$ & 0.48 & $2 \%$ \\
\hline $1000 / 1000-3000$ & 0.68 & $7 \%$ \\
\hline $5000 / 100-1000$ & 0.48 & $15 \%$ \\
\hline $5000 / 100-1000$ & 0.68 & $8 \%$ \\
\hline $5000 / 1000-3000$ & 0.48 & $1 \%$ \\
\hline $5000 / 1000-3000$ & 0.68 & $6 \%$ \\
\hline
\end{tabular}

Mirroring the results showed in Section 3.2, the error increases when the burst size is small. However, the error is smaller in this case than it is in the high-performance cluster case because the bandwidth is lower and the variation in its range is shorter. Also, the transference of short bursts in the workstation network environment is more sensitive to bandwidth variation than the transference of large bursts. This is shown in Table 7, which shows the slowdown produced with a benchmark that measures the bandwidth using a specific burst size. For short bursts, the slowdown is approximately 5, whereas for larger bursts it is approximately 2 (assuming the same load in the network). As for high-performance clusters, a benchmark that calculates the bandwidth in the workstation network using a tailored burst size overcomes this problem.

Table 7: Experiments with Bandwidth Benchmarks

\begin{tabular}{|c|c|}
\hline $\begin{array}{c}\text { Burst size (number of messages / } \\
\text { message size in words) used by the } \\
\text { Benchmark }\end{array}$ & $s d_{a \rightarrow b}$ \\
\hline \hline $10 / 100$ & 5.09 \\
\hline $10 / 500$ & 2.26 \\
\hline $1000 / 500$ & 2.06 \\
\hline $5000 / 500$ & 2.05 \\
\hline
\end{tabular}

\section{Evaluation of the Model}

The models developed for slowdown on computation were based on knowledge of the relative amounts of computation and communication for each application. We assume that the time to compute is longer than the computation/communication cycles of the competing applications and will be affected by both computation and communication activities. If the targeted computation is fast in comparison with the duration of these cycles, i.e., the time to compute is close to the duration of one computation/communication cycle, the accuracy of the model decreases. The error may also increase if the applications exhibit a non-random behavior, e.g., if the computation and communication cycles of the competing applications are either totally synchronized or totally nonsynchronized with one another.

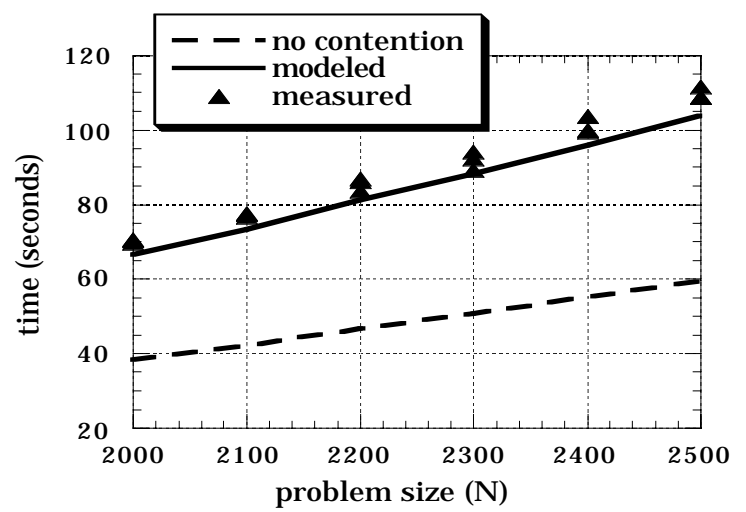

Figure 11: Time to execute the SOR benchmark in dedicated mode and competing with one application that has a 24.2-second computation/communication cycle.

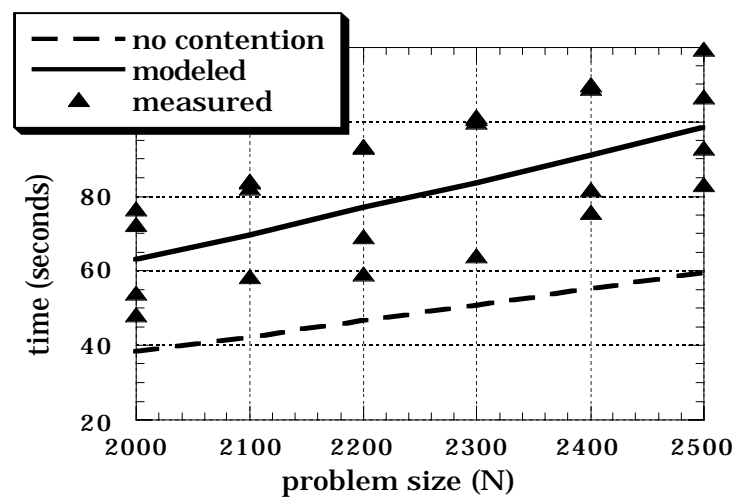

Figure 12: Time to execute the SOR benchmark in dedicated mode and competing with one application that has a 288.0-second computation/communication cycle.

Figure 11 and Figure 12 illustrate the difference in accuracy obtained in the prediction of the time to execute the SOR benchmark serially on a DEC Alpha in two settings. In both settings, the SOR competes for the CPU with one application that alternates computation with communication. In Figure 11, the time to execute one computation/communication cycle of the competing application was 24.2 seconds. In this case, the time to execute the algorithm was longer than one computation/ communication cycle of the competing application, and the average error was 5\%. In Figure 12, the time to 
execute one computation/communication cycle of the competing application was 288.0 seconds. In this case, the time to execute the same algorithm was shorter than one computation/communication cycle of the competing application, and the variation of the actual times to execute the algorithm causes the average error to expand to within $17 \%$.

\section{Summary and Future Work}

Most applications share the resources of networked environments with other applications. Since system load can vary dramatically, allocation strategies that assume that resources have a constant availability and/or capability are unlikely to promote performance-efficient allocations in practice. It is critical to provide a realistic model of the effects of contention on application performance in order to best allocate application tasks to machines.

In this paper, we have presented a model that predicts contention effects in representative networked platforms. The model provides a basis for predicting realistic communication and computation costs. Note that the variation in times to execute applications on production systems is typically high, and therefore makes it difficult for a contention model to be accurate. For this reason, our objective has been to obtain accuracy on an average basis. The experiments executed thus far have shown that we have achieved our goal.

We are continuing to investigate causes for contention. We are currently extending our model to include memory constraints, as well as priority issues. We also plan on characterizing the setting in which contending applications execute for only part of the execution of a given application. Since system load may vary during the execution of an application, the slowdown factors should be recalculated when the job mix changes, and task migration should be considered.

Finally, the slowdown factors developed for these platforms can be used for general networked systems. We believe that an accurate contention model is a fundamental part of a performance-efficient allocation strategy for networked systems and, ultimately, essential to the use of these systems as platforms for parallel and/or heterogeneous applications.

\section{Acknowledgments}

We would like to thank our colleagues in the UCSD Parallel Computation Laboratory and in the San Diego Supercomputer Center for their support, specially Stephen Fink, Mike Vildibill, Ken Steube, Cindy Zheng, and Victor Hazlewood. We would also like to thank Professor Joseph Pasquale for the usage of the machines in the Computer Systems Laboratory at UCSD.

\section{References}

[1] M. Atallah, C. Black, D. Marinescu, H. Siegel, and T. Casavant, "Models and Algorithms for Coscheduling ComputeIntensive Tasks on a Network of Workstations", Journal of Parallel and Distributed Computing, vol. 16, pp. 319-327, 1992.

[2] A. Beguelin, J. Dongarra, G. Geist, R. Manchek, and V. Sunderam, "Graphical Development Tools for NetworkBased Concurrent Supercomputing", Proceedings of Supercomputing 91, pp. 435-444.

[3] F. Berman, R. Wolski, S. Figueira, J. Schopf, and G. Shao, "Application-Level Scheduling on Distributed Heterogeneous Networks", in Proceedings of Supercomputing'96, November 1996.

[4] A. Bricker, M. Litzkow, and M. Livny, "Condor Technical Summary", Technical Report \#1069, University of Wisconsin, Computer Science Department, May 1992.

[5] W. L. Briggs, "A Multigrid Tutorial”, Society for Industrial and Applied Mathematics, Philadelphia, Pennsylvania, 1987.

[6] H. Dietz, W. Cohen, and B. Grant, "Would you run it here...or there? (AHS: Automatic Heterogeneous Supercomputing)", Proceedings of the International Conference on Parallel Processing, vol. II, pp. 217-221, August 1993.

[7] A. C. Dusseau, R. H. Arpaci, and D. E. Culler, "Effective Distributed Scheduling of Parallel Workloads, in Proceedings of ACM SIGMETRICS'96, pp. 25-36, May 1996.

[8] S. M. Figueira and F. Berman, "Modeling the Effects of Contention on the Performance of Heterogeneous Applications," in Proceedings of the Fifth IEEE International Symposium on High Performance Distributed Computing, August 1996.

[9] S. J. Fink, S. B. Baden, and S. R. Kohn, "Flexible Communication Mechanisms for Dynamic Structured Applications", in Proceedings of the Third International Workshop on Parallel Algorithms for Irregularly Structured Problems, Santa Barbara, CA, August 1996.

[10] S. Leutenegger and X. Sun, "Distributed Computing Feasibility in a Non-Dedicated Homogeneous Distributed System”, NASA - ICASE Technical Report 93-65, September 1993.

[11] Matrix Multiply, http://HTTP.CS.Berkeley.EDU/ demmel/ $\operatorname{cs} 267$.

[12] Message-Passing Interface Forum, "MPI: A Message-Passing Interface Standard", University of Tennessee, Knoxville, TN, June 1995.

[13] V. Sunderam, "PVM: A Framework for Parallel Distributed Computing", Concurrency: Practice and Experience, vol. 2, n. 4, pp. 315-339, December 1990.

[14] R. Wolski, "Dynamically Forecasting Network Performance Using the Network Weather Service," UCSD CS Technical Report \#CS96-494.

[15] X. Zhang and Y. Yan, "A Framework of Performance Prediction of Parallel Computing on Non-dedicated Heterogeneous Networks of Workstations", Proceedings of 1995 International Conference of Parallel Processing, vol. I, pp. 163-167, 1995. 